\title{
Hysteresis effects in rotating Bose-Einstein condensates
}

\author{
B. Jackson ${ }^{1,2}$ and C. F. Barenghi ${ }^{2}$ \\ ${ }^{1}$ Dipartimento di Fisica, Università di Trento and CNR-INFM \\ REDD Center on Bose-Einstein Condensation, I-38050 Povo, Italy \\ ${ }^{2}$ School of Mathematics and Statistics, \\ University of Newcastle upon Tyne, NE1 7RU, United Kingdom
}

(Dated: October 21, 2018)

\begin{abstract}
We study the formation of vortices in a dilute Bose-Einstein condensate confined in a rotating anisotropic trap. We find that the number of vortices and angular momentum attained by the condensate depends upon the rotation history of the trap and on the number of vortices present in the condensate initially. A simplified model based on hydrodynamic equations is developed, and used to explain this effect in terms of a shift in the resonance frequency of the quadrupole mode of the condensate in the presence of a vortex lattice. Differences between the spin-up and spin-down response of the condensate are found, demonstrating hysteresis phenomena in this system.
\end{abstract}

PACS numbers: 03.75.Lm, 03.75.Kk, 67.40.Vs 


\section{INTRODUCTION}

The realization of Bose-Einstein condensation (BEC) in ultracold gases has provided a powerful new system in the study of superfluids, combining better experimental control and theoretical tractability than liquid helium. A particularly striking example concerns the response of the fluid to rotation. Due to the irrotationality property of superfluids, application of a rotating perturbation results in the creation of a lattice of quantized vortex lines, a process that was originally observed in He-II [1]. Analogous experiments in ultracold gases have found the formation of similar structures in Bose condensates [2, 3, 44, 5, 6, 7, 8], and recently in a fermionic gas that is superfluid due to Cooper pairing between atoms [9].

An interesting question raised by these experiments concerns the angular velocity of the rotating potential required to nucleate vortices. Since the energy per particle of a condensate containing a vortex, $E_{v}$, exceeds that of the ground state, $E_{0}$, by transforming to a frame rotating with angular velocity $\Omega$ it is straightforward to show that vortices are energetically favored if $\Omega>\left(E_{v}-E_{0}\right) / \hbar[10]$. However, higher rotation rates are generally required to nucleate vortices in experiments [2, 5, 6, 8], which reflects the existence of an energy barrier to vortex formation [11]. This can be overcome by excitation of collective modes localized at the surface of the condensate [12, 13].

The presence of two different critical angular velocities implies the existence of metastable states, where vortices are energetically favorable but dynamically are unable to form, and in turn the possibility of hysteresis phenomena [12, 14]. Hysteresis has long been observed in rotating superfluid helium. For example, experiments have found a significant difference between the number of vortices obtained when the fluid is spun up to when it is spun down [15]. Reproducible hysteresis loops were measured by minimizing the mechanical and thermal noise of the apparatus [16]. Jones et al. [17] attempted to explain this effect by using a principle of local momentum conservation. Fortunately atomic Bose-Einstein condensates are less noisily coupled to their environment than rotating liquid helium, and, unlike liquid helium, can be studied using a model that has great predictive power, namely the Gross-Pitaevskii (GP) equation.

In this article we discuss a particular example of hysteresis, where the amount of circulation already present in a Bose condensate influences the final angular momentum and number of vortices that can be attained. This effect has been exploited in the experiment of 
Ref. 18] to rotate the condensate to high angular velocities. The aim of this paper is to explore this issue theoretically in more detail. Following previous studies [19, 20, 21, 22, 23, 24] we solve the GP equation for the condensate wavefunction $\Psi(\boldsymbol{r}, t)$ to study the vortex formation process in a rotating anisotropic trap. However, we go further by considering cases where the trap rotation frequency is changed during the simulation, as well as when vortices are already present in the condensate. Moreover, new insight is gained by comparing our findings to the results of a hydrodynamic model 25] of the condensate, which yields a resonance in the angular momentum transfer associated with excitation of the quadrupole collective mode. The presence of a vortex lattice then has the effect of shifting this mode frequency and therefore the resonance to higher values. As a further example of hysteresis we consider the case where the trap rotation frequency is slowly ramped up and then down, demonstrating a difference in the trajectories of the angular momentum between the two cases.

We note that the discussion in this paper is restricted to very low temperatures, where the thermal cloud is insignificant and the condensate dynamics can be accurately modelled with the GP equation. An interesting issue concerns the effect of finite temperatures on the phenomena described here. However, to address this question requires one to treat the dynamics of both the condensate and thermal cloud consistently, since the thermal cloud also responds to a rotating potential by spinning up during a timescale related to the frequency of collisions between the atoms [26]. Treatment of the coupled dynamics is conceptually rich and numerically intensive, and is outside the scope of this work.

\section{VORTEX FORMATION}

The GP equation is solved numerically for a harmonically trapped condensate in 2D, which corresponds to the case where the axial trap frequency is much larger than the mean field interaction energy, such that motion along the axial direction is frozen. Similar 2D studies [20, 24] have previously found good qualitative, and in some cases quantitative, agreement with experimental data even when this condition is not satisfied [2, 6], confirming that much of the crucial physics of the vortex formation process is captured in $2 \mathrm{D}$.

Angular momentum is imparted to the condensate by rotating an elliptically-deformed 
harmonic trap, which is represented by the potential

$$
V(\boldsymbol{r})=\frac{1}{2} m \omega_{\perp}^{2}\left[(1+\epsilon) x^{\prime 2}+(1-\epsilon) y^{\prime 2}\right]
$$

where a rotational transformation of the $(x, y, z)$ Cartesian coordinate system is used, such that $x^{\prime}=x \cos (\Omega t)+y \sin (\Omega t)$ and $y^{\prime}=-x \sin (\Omega t)+y \cos (\Omega t)$ correspond to rotation of the trap at frequency $\Omega$. Dimensionless units are also used, with the units of length, time and energy given by $\left(\hbar /\left(m \omega_{\perp}\right)\right)^{1 / 2}, \omega_{\perp}^{-1}$ and $\hbar \omega_{\perp}$ respectively. The GP equation then becomes

$$
i \frac{\partial}{\partial t} \Psi=\left\{\frac{1}{2}\left[-\nabla^{2}+(1+\epsilon) x^{2}+(1-\epsilon) y^{\prime 2}\right]+g|\Psi|^{2}\right\} \Psi .
$$

Mean field interactions are represented by $g=4 \pi N^{\prime} a$, where $N^{\prime}$ is the number of atoms per unit length in the axial $z$ direction, while $a$ denotes the $s$-wave scattering length. Throughout this paper we will use $g=450$, although the results are expected to be generally applicable to other interaction strengths.

To provide the initial condition for the simulation, Eq. (2) is numerically propagated in imaginary time with $\epsilon=0$ and $\Omega=0$, such that the wavefunction converges to the condensate ground state without vortices. In order to model vortex formation the simulation is then run in real time with a rotating elliptical trap $(\epsilon=0.1, \Omega>0)$, where the deformation $\epsilon$ is switched on abruptly at $t=0$, rather than being turned on gradually.

Fig. 11 illustrates the subsequent time-dependent response of the condensate by plotting the mean angular momentum $\left\langle L_{z}\right\rangle=\int d \boldsymbol{r} \Psi^{*} \hat{L}_{z} \Psi$, with $\hat{L}_{z}=i\left[y \partial_{x}-x \partial_{y}\right]$. If $\Omega=0.78$ (curve a) one sees that the angular momentum increases initially, undergoing large oscillations before settling to an almost constant value for $t>250$, similar to behavior found in previous studies [20, 23, 24] as well as experiments [8]. The initial oscillations correspond to variations in the quadrupolar deformation of the condensate which subsequently diminish as vortices enter the condensate. The vortices then continue to undergo complicated dynamics, albeit in such a way that the angular momentum remains in quasi-equilibrium. However, the vortices do not crystallize to form a stable, ordered lattice, which would require the inclusion of dissipation [21, 22, 27, 28] or integration of the GP equation over much longer timescales [23, 24].

If the trap rotation rate is significantly smaller or larger than $\Omega=0.78$ then the condensate angular momentum displays a periodic time dependence (see Fig. 1 curve b, for $\Omega=0.9$ ). This behavior corresponds to an oscillation of the quadrupolar deformation of the 


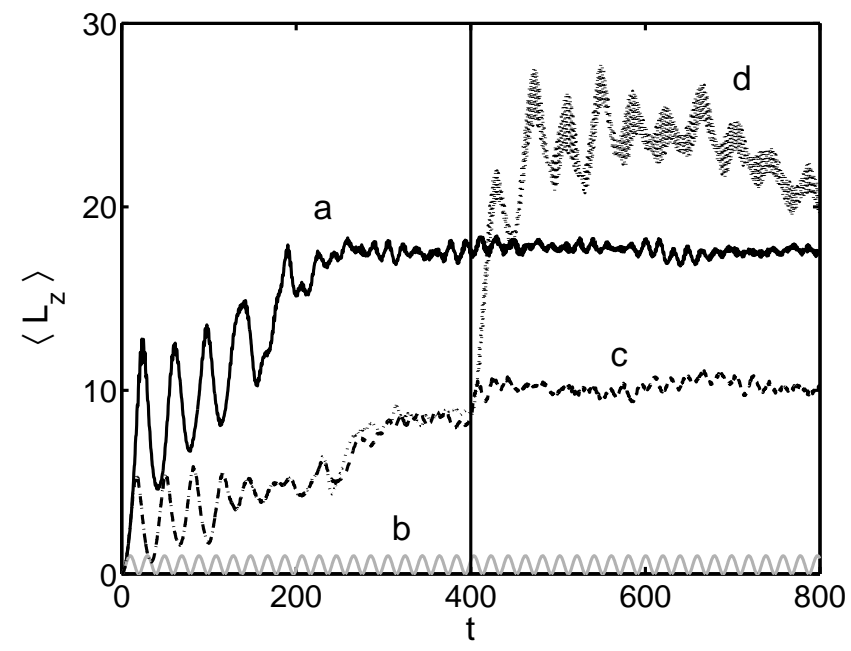

FIG. 1: Angular velocity, $\left\langle L_{z}\right\rangle$ (in units of $\hbar$ ) as a function of time, $t$ (units of $\omega_{\perp}^{-1}$ ) for $\epsilon=0.1$ and no vortices present in the condensate initially. The solid lines plot the results when the initial trap rotation frequency $\Omega$ (in units of $\omega_{\perp}$ ) is maintained up to $t=800$, with $\Omega=0.78$ (curve a, black) and $\Omega=0.9$ (curve b, grey). The broken lines (curves c and d) represent cases where $\Omega=0.7$ up to $t=400$ (marked by a vertical line), after which the rotation frequency is changed to $\Omega=0.78$ (c, dashed) or $\Omega=0.9$ (d, dotted). Note that all of the figures in this paper are plotted in dimensionless units.

condensate, $\delta=\left\langle y^{\prime 2}-x^{\prime 2}\right\rangle /\left\langle y^{\prime 2}+x^{\prime 2}\right\rangle$. The angular momentum is found to be close to the value $\left\langle L_{z}\right\rangle=\Omega \Theta$ if the momentum of inertia is given by the expression for a irrotational superfluid, $\Theta=\delta^{2}\left\langle x^{\prime 2}+y^{\prime 2}\right\rangle$ [29, 30]. Hence the condensate remains irrotational and no vortices are nucleated, in contrast to the case where $\Omega=0.78$. Note that the oscillations in the quadrupolar deformation arise due to the sudden imposition of the rotating trap's deformation, and their amplitude is lower if $\epsilon$ is turned on gradually.

In the two examples given, the trap rotation rate was maintained at a constant value throughout the simulation. If, instead, the rotation rate is changed to another value midway through the run, then very different results are found. Curves c and d of Fig. 1 represent simulations where for $0<t<400$ the trap rotates at $\Omega=0.7$, resulting in vortex nucleation. At $t=400$ the rotation rate is abruptly changed to either $\Omega=0.78$ (curve c) or $\Omega=0.9$ (curve d). In the former case the angular momentum increases only slightly, and remains much smaller $\left(\left\langle L_{z}\right\rangle \simeq 10\right)$ than in the case where $\Omega=0.78$ from the beginning $\left(\left\langle L_{z}\right\rangle \simeq 18\right)$. For $\Omega=0.9$ (curve d), however, the angular momentum jumps to a much larger value 
(corresponding to a major increase in the number of vortices), which is in stark contrast with the lack of vortices when the condensate is rotated at this frequency initially (curve b). These examples demonstrate that the vortex formation process strongly depends upon the "rotation history" of the condensate.

To explore this question further, we have also conducted simulations where the trap rotation frequency remains constant throughout each run, but the initial state already contains vortices. These states can be obtained by solving the GP equation for a non-inertial frame of reference which rotates at constant angular velocity $\Omega_{0}$, where $t$ is now imaginary

$$
i \frac{\partial}{\partial t} \Psi=\left[\frac{1}{2}\left(-\nabla^{2}+x^{2}+y^{2}\right)+g|\Psi|^{2}-\Omega_{0} \hat{L}_{z}\right] \Psi
$$

Hereafter we will use $\Omega_{0}$ to distinguish the rotation of the reference frame (in which we solve the GP equation in imaginary time) from the (real time) trap rotation rate $\Omega$. For sufficiently large $\Omega_{0}$, imaginary time propagation leads to the appearance of vortices, which eventually settle into an ordered lattice to yield the stationary solution for this rotating frame.

The wavefunction thus found can be used as the initial condition for Eq. (2), which is integrated in real time as in the previous section. Fig. 2 shows the resulting time evolution of the angular momentum with $\Omega=0.78$ for different initial states. The black solid line (curve e) corresponds to the same run represented by curve a in Fig. 1, where the condensate contains no vortices initially $\left(\Omega_{0}=0\right)$. The grey line (curve $\mathrm{f}$ ), by way of contrast, is for $\Omega_{0}=0.5$, where the initial state contains six vortices and possesses an angular momentum of $\left\langle L_{z}\right\rangle=3.64$. The subsequent rotation of the trap at $\Omega=0.78$ leads to a final angular momentum of $\left\langle L_{z}\right\rangle \simeq 11$, which is only around $60 \%$ of the value attained when no vortices are present initially. If higher values of $\Omega_{0}$ (corresponding to more initial vortices) are used, this deficit in the angular momentum becomes even larger. Indeed, if $\Omega_{0}=0.78$, the angular momentum remains almost constant throughout the evolution in real time.

These differences in angular momentum reflect similar variations in the number of vortices present in the condensate. This is illustrated in Fig. 3, where (a)-(c) show snapshots of the condensate density, $|\Psi(\boldsymbol{r}, t)|^{2}$, at various times for $\Omega_{0}=0$ and $\Omega=0.78$. The corresponding case for $\Omega_{0}=0.6$ is shown in Figs. [3 (d)-(f); vortices are already present initially, but the final number of vortices at $t=400$ is less than for $\Omega_{0}=0$ (comparing Figs. 3 (c) and (f)).

To help explain these differences, in Fig. 4 we plot the angular momentum as a function 


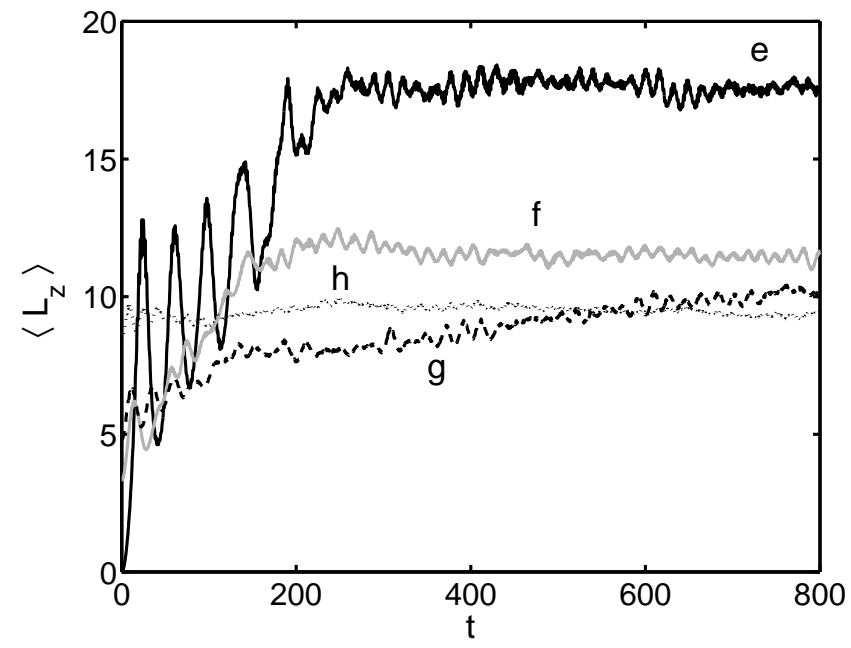

FIG. 2: Angular momentum as a function of time for a condensate in a rotating trap with $\epsilon=$ 0.1 and $\Omega=0.78$. The various simulations use different initial conditions, which correspond to stationary states for an isotropic condensate in a frame rotating with angular velocity $\Omega_{0}$. Plotted are $\Omega_{0}=0$ (curve e, solid black line), $\Omega_{0}=0.5$ (curve f, grey), $\Omega_{0}=0.6$ (curve g, dashed) and $\Omega_{0}=0.78$ (curve $\mathrm{h}$, dotted).
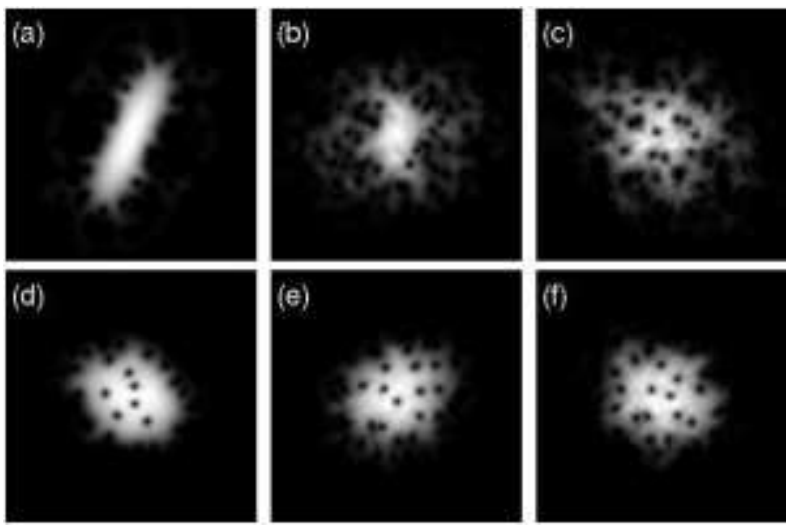

FIG. 3: Condensate density $|\Psi(\boldsymbol{r}, t)|^{2}$ with a trap rotating at angular velocity $\Omega=0.78$ for (a) $t=100$, (b) $t=200$, and (c) $t=400$, with $\Omega_{0}=0$. Panels (d)-(f) show a similar simulation but with $\Omega_{0}=0.6$, for (d) $t=50$, (e) $t=200$, (f) $t=400$. The holes in the density profiles correspond to vortices.

of the trap rotation frequency for $\Omega_{0}=0$ and $\Omega_{0}=0.5$. If there are no vortices and $\left\langle L_{z}\right\rangle$ has a periodic time dependence then the plotted value is the peak value between $t=0$ and $t=400$. If vortices are present the value of $\left\langle L_{z}\right\rangle$ is taken at $t=400$, at which 
point the angular momentum has generally plateaued at an approximately constant value. The filled circles represent the results for the initial condition $\Omega_{0}=0$, and it is apparent that appreciable vortex formation (and hence angular momentum transfer) occurs in the approximate range $0.68<\Omega<0.88$. Furthermore, within this range the angular momentum rises with increasing $\Omega$, in agreement with previous experimental [6] and theoretical 24] studies, where a similar dependence was found when plotting the final number of vortices against $\Omega$.

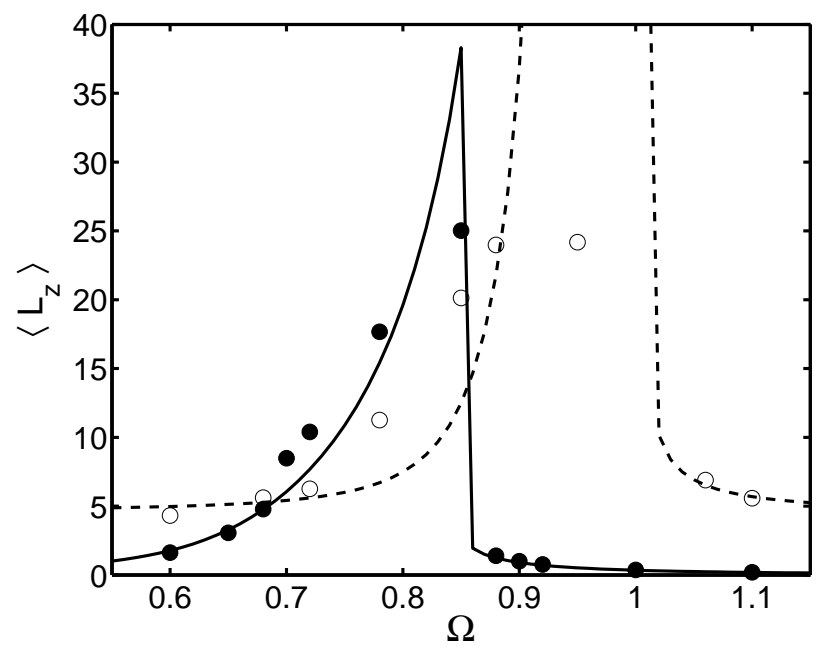

FIG. 4: Maximum angular momentum attained by a condensate in an elliptical trap rotating with frequency $\Omega(\epsilon=0.1)$. The solid and open circles show the results for $\Omega_{0}=0$ and $\Omega_{0}=0.5$ respectively. The lines plot the maximum angular momentum found by solving the hydrodynamic model (8] for $\Omega_{0}=0$ (solid) and $\Omega_{0}=0.5$ (dashed).

The angular momenta attained at $t=400$ when the initial condition is $\Omega_{0}=0.5$ are plotted in Fig. 4 with open circles [31]. We see that, compared to the $\Omega_{0}=0$ case, appreciable increases in $\left\langle L_{z}\right\rangle$ tend to take place at higher $\Omega$. Hence, nucleation of additional vortices occurs at higher rotation frequencies when vortices are already present in the condensate. This effect accounts for the differences in angular momentum apparent in Figs. 1 and 2.

\section{HYDRODYNAMIC MODEL}

The numerical solutions of the Gross-Pitaevskii equation discussed in the previous section accurately treat the dynamics of the condensate at very low temperatures. However, it is 
also instructive to consider a simplified, approximate model that is much less numerically intensive to solve than the GP equation. This allows us to more easily explore parameter space, as well as providing new insight into the phenomena observed so far. Our simplified model is based upon the equations of rotational hydrodynamics, which provide a description of the condensate in the Thomas-Fermi (large $g$ ) regime

$$
\begin{gathered}
\frac{\partial n}{\partial t}+\nabla \cdot(n \boldsymbol{v})=0 \\
\frac{\partial \boldsymbol{v}}{\partial t}+(\boldsymbol{v} \cdot \nabla) \boldsymbol{v}+\nabla(V+g n)=0 .
\end{gathered}
$$

Following [25] we solve Eqs. (44) and (51) to describe the dynamics by employing the following ansatz for the density and velocity 33$]$

$$
\begin{gathered}
n(\boldsymbol{r})=a_{0}+a_{x} x^{2}+a_{y} y^{2}+a_{x y} x y, \\
\boldsymbol{v}(\boldsymbol{r})=\mathbf{\Omega}_{\mathbf{0}} \times \boldsymbol{r}+\nabla\left(b_{x} x^{2}+b_{y} y^{2}+b_{x y} x y\right),
\end{gathered}
$$

where $a_{i}, b_{i}$ and $\Omega_{\mathbf{0}}$ are time-dependent parameters. Note that the velocity (71) includes both rotational and irrotational components, where the former assumes that the combined velocity field of the vortices in the lattice approximates a solid body rotation. Substituting (6) and (7) into (4) and (5) yields a set of differential equations for each parameter, which are integrated in time using a fourth-order Runge-Kutta scheme [34]

$$
\begin{aligned}
\dot{a}_{0}+2 a_{0}\left(b_{x}+b_{y}\right) & =0, \\
\dot{a}_{x}+\Omega_{0} a_{x y}+6 a_{x} b_{x}+2 a_{x} b_{x}+a_{x y} b_{x y} & =0, \\
\dot{a}_{y}-\Omega_{0} a_{x y}+2 a_{y} b_{x}+6 a_{y} b_{y}+a_{x y} b_{x y} & =0, \\
\dot{a}_{x y}-2 \Omega_{0} a_{x}+2 \Omega_{0} a_{y}+4 a_{x y}\left(b_{x}+b_{y}\right)+2\left(a_{x}+a_{y}\right) b_{x y} & =0, \\
\dot{b}_{x}+\frac{1}{2}\left(4 b_{x}^{2}-\Omega_{0}^{2}+b_{x y}^{2}+1+\epsilon_{x}+2 g a_{x}\right) & =0, \\
\dot{b}_{y}+\frac{1}{2}\left(4 b_{y}^{2}-\Omega_{0}^{2}+b_{x y}^{2}+1-\epsilon_{x}+2 g a_{y}\right) & =0, \\
\dot{b}_{x y}+2\left(b_{x}+b_{y}\right) b_{x y}+\epsilon_{y}+g a_{x y} & =0, \\
\dot{\Omega}_{0}+2\left(b_{x}+b_{y}\right) \Omega_{0} & =0,
\end{aligned}
$$

where $\epsilon_{x}=\epsilon \cos (2 \Omega t)$ and $\epsilon_{y}=\epsilon \sin (2 \Omega t)$, with $\Omega$ the trap rotation frequency. Once the time evolution of these properties are known quantities such as the angular momentum can be calculated by integration of (6) and (77). 
The solution of Eqs. (8, 15) for a rotating elliptical trap yields an angular momentum that oscillates in time, similar to the behavior shown in curve b of Fig. 1. The peak angular momenta as a function of $\Omega$ are plotted in Fig. 5 for various values of $\epsilon$ and $\Omega_{0}=0$. For $\epsilon=0.001$ the response is symmetrical about a peak near $\Omega=1 / \sqrt{2}$. This sharp resonance arises since a perturbation rotating with frequency $\Omega$ is resonant with a surface mode with azimuthal quantum number $m$ when $\omega-m \Omega \simeq 0$, so that for a quadrupolar trap deformation the $m=+2$ mode should be resonantly excited when $\Omega_{\text {res }} \simeq \omega_{+2} / 2[12$. With increasing $\epsilon$ the resonance becomes higher and wider as well as more asymmetric, until at $\epsilon=0.1$ there is a steep downward gradient immediately following the peak. Note that the resonance peaks are symmetric when Eqs. (8] 15) are linearized for small departures from equilibrium, demonstrating that their asymmetry is a consequence of the nonlinearity of the equations.

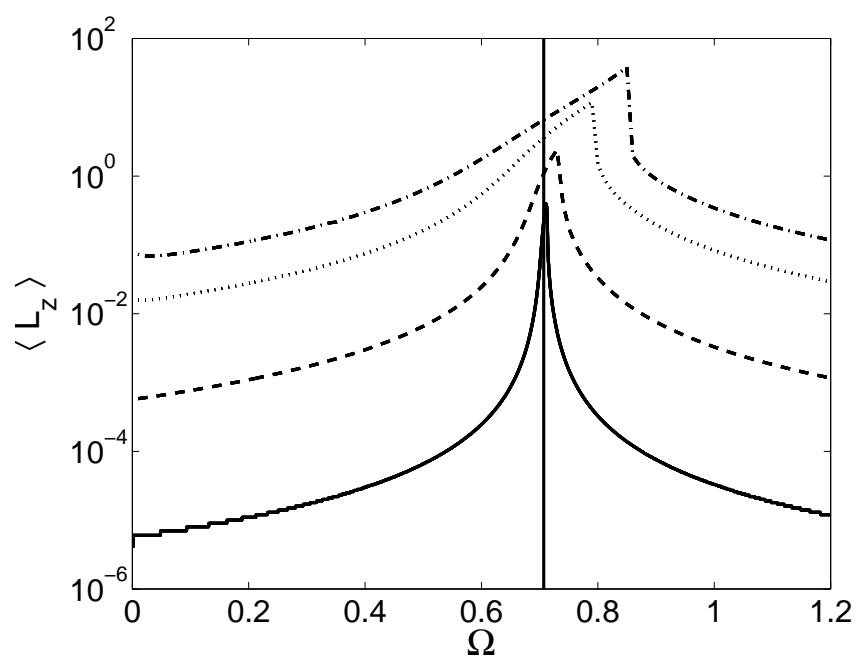

FIG. 5: Maximum angular momentum $\left\langle L_{z}\right\rangle$ as a function of trap rotation frequency, $\Omega$, solving Eqs. (8] 15) for $\Omega_{0}=0$. The different curves show results for $\epsilon=0.001$ (solid), $\epsilon=0.01$ (dashed), $\epsilon=0.05$ (dotted), and $\epsilon=0.1$ (dot-dashed). The vertical line marks $\Omega=1 / \sqrt{2}$.

The results of the hydrodynamic model are compared to those of the GP equation in Fig. 4. The solid line plots the maximum angular momentum as a function of $\Omega$ for $\Omega_{0}=0$, where one sees a close agreement with the GP results. Such correspondence is pleasing, but perhaps to be anticipated when vortices are not nucleated, since Eqs. (6) and (17) are expected to be good approximations and the time dependence of $\left\langle L_{z}\right\rangle$ is oscillatory in both cases. The agreement is more surprising in the region $0.68<\Omega<0.88$, when vortices are nucleated and the time dependence departs from oscillatory behavior. It appears that 
the final, steady angular momentum attained is still close to the peak amplitude of the oscillations in the absence of vortices.

The maximum $\left\langle L_{z}\right\rangle$ for $\Omega_{0}=0.5$ is plotted with the dashed line in Fig. 4 . The agreement with the GP results is again relatively good, even though the approximation of solid-body rotation in (7) is not expected to be particularly accurate for the small number of vortices present in this case. One sees a shifting of the resonance to higher trap rotation frequencies for the larger $\Omega_{0}$, which is related to changes in the frequencies of the quadrupole collective modes. By linearizing (8] 15) for small amplitude oscillations around $\epsilon=0$ one finds these frequencies to be [25]

$$
\omega_{ \pm 2}=\sqrt{2-\Omega_{0}^{2}} \pm \Omega_{0}
$$

This expression provides a simple and useful way to understand the behavior of quadrupole mode frequencies with changing rotation. In particular, one sees that the counterpropagating $m=+2$ and $m=-2$ quadrupole modes are degenerate at $\omega=\sqrt{2}$ for $\Omega_{0}=0$, while in the presence of a vortex lattice this degeneracy is broken such that $\omega_{+2} \rightarrow 2$ and $\omega_{-2} \rightarrow 0$ for $\Omega_{0} \rightarrow 1$. This general behavior of the $m=+2$ frequency increasing with $\Omega_{0}$ is reflected in Fig. 4, albeit modified to account for the nonlinearity introduced with $\epsilon \neq 0$.

A further consequence of this argument is that to remove angular momentum, and therefore vortices, from the condensate requires the trap to be rotating in the opposite direction with an angular velocity approximately half that of the $m=-2$ mode frequency. We have confirmed this by performing simulations with $\Omega_{0}=0.5$ and $\Omega \leq 0$, and the results are presented in Fig. 6. For $\Omega=-0.3$ the angular momentum undergoes small oscillations and remains close to the original value $\left\langle L_{z}\right\rangle \simeq 3.64$, since this trap rotation rate is sufficiently far from the resonance. This is also found to be true when $\Omega=-0.8$, albeit with smaller amplitude oscillations due to being further from resonance. However, when $\Omega=-0.5$ the angular momentum rapidly decreases and becomes negative as the vortices that are originally present leave the condensate and vortices of opposite sign enter, eventually settling into a quasi-equilibrium state. The inset of Fig. 6] compares the minimum of $\left\langle L_{z}\right\rangle$ in the three simulations to the result of solving the hydrodynamic model (8) [15) under the same conditions, once again demonstrating reasonable agreement between the two approaches. As expected, the peak of the resonance is close to $-\omega_{-2} / 2$, where $\omega_{-2} \simeq 0.82$ from (16), although as before it is skewed towards higher $|\Omega|$ due to the large value of $\epsilon$. 


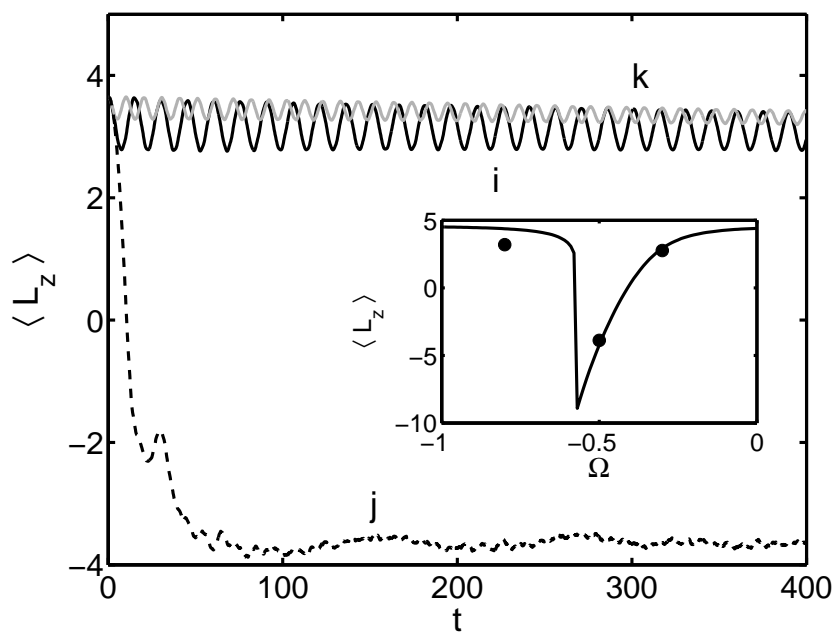

FIG. 6: Angular momentum as a function of time for $\Omega_{0}=0.5$, and $\Omega=-0.3$ (curve i, solid black line), $\Omega=-0.5$ ( $\mathrm{j}$, dashed), and $\Omega=-0.8$ ( $\mathrm{k}$, grey). Inset: Minimum angular momentum for different $\Omega$ from GP simulations (points), compared to (solid line) the results of solving Eqs. (815) for $\Omega_{0}=0.5$.

\section{HYSTERESIS DURING LINEAR RAMPING}

Finally, we again use numerical simulations of the GP equation (2) to study the difference in the response of the condensate between when it is spun up to when it is spun down. We begin with an initial condition containing vortices, such that the wavefunction is a stationary solution of Eq. (3) with $\Omega_{0}=0.5$. An elliptically-deformed trap potential $(\epsilon=0.1)$ is then rotated with a time-dependent angular velocity of the following form

$$
\Omega(t)= \begin{cases}0.5+3.75 \times 10^{-4} t & \text { if } 0 \leq t \leq 400 \\ 0.8-3.75 \times 10^{-4} t & \text { if } 400<t \leq 800\end{cases}
$$

Hence during the first half of the simulation $\Omega$ is linearly ramped up from 0.5 to 0.65 , while in the second half it is ramped down from 0.65 to 0.5 . These correspond to "spin-up" and "spin-down" experiments respectively.

The result is shown in Fig. [7, where the angular momentum is plotted against both $\Omega$ (lower abscissa) and time (upper abscissa). In turn, on the upper abscissa times are shown for both the spin-up (black text) and spin-down (grey text) processes, where the corresponding plotted curves are represented by the same colors. During the spin-up process $\left\langle L_{z}\right\rangle$ does not increase initially, but undergoes oscillatory behavior. This corresponds to the left side of 


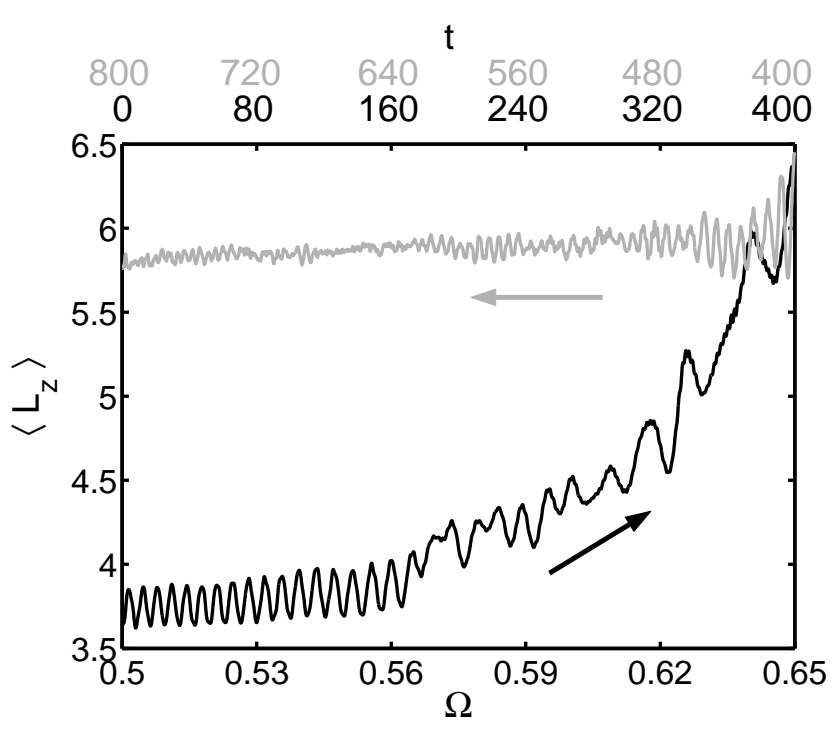

FIG. 7: Hysteresis in the angular momentum, $\left\langle L_{z}\right\rangle$, of the condensate wavefunction, initially a stationary solution of the GP equation (3) with $\Omega_{0}=0.5$. The angular velocity of a rotating elliptical trap $(\epsilon=0.1)$ follows Eq. (17). It is linearly ramped from $\Omega=0.5$ at $t=0$ to $\Omega=0.65$ at $t=400$ (black line), and subsequently for $t>400$, is linearly ramped down such that $\Omega=0.5$ at $t=800$ (grey line). Note that the abscissa is labelled with $\Omega$ (bottom) and $t$ (top), where the latter has two different tick labels for spin-up (black) and spin-down (grey).

Fig. 4. where the rotation frequency is far from the $m=+2$ quadrupolar resonance. Then, as $\Omega$ approaches resonance angular momentum is transferred to the condensate, until $\left\langle L_{z}\right\rangle \simeq 6$ at $t=400$. During the subsequent spin-down process, however, the angular momentum does not follow the path of the spin-up, but remains almost constant with only a slight decrease at the end. This reflects the fact that the rotation rate is far from being resonant with the $m=-2$ mode, the excitation of which is required to remove angular momentum from the system. Once again this clearly illustrates the importance of the rotation history on the attained angular momentum, and hence demonstrates the possibility of observing hysteresis phenomena in rotating condensates.

Finally, we should note that hysteresis behavior was also found by García-Ripoll and Pérez-García in Ref. 14]. A major difference with respect to the present work is that in [14] the time-independent GP equation was solved to find the stationary solutions for different rotation rates, while here the dynamics are considered explicitly by solving the timedependent GP equation. Moreover, the results of Ref. 14] are based on the assumption that 
all collective modes are excited, and vortex formation occurs when the rotation frequency $\Omega$ first becomes resonant with one of the modes. In experiments this would correspond to relatively high temperatures where the collective excitations are thermally populated to an appreciable extent and the thermal cloud is in rotation (similarly to the experiment of Haljan et al. 7]). In contrast, we have been interested in the case where vortex nucleation is induced by a rotating elliptical trap, so that the quadrupole mode is predominately excited and becomes unstable prior to vortex nucleation.

\section{CONCLUSIONS}

In summary, we have studied the process of vortex formation in a rotating dilute BoseEinstein condensate using numerical simulations of the Gross-Pitaevskii equation. We have paid particular attention to the amount of angular momentum transferred to the condensate for different rotation angular velocities of an elliptically-deformed trapping potential. The angular momentum of the condensate (and hence the number of vortices) not only depends upon the final rotation rate of the trap, but also upon the history of the rotation. Specifically, by initially rotating at one angular velocity, $\Omega$, until vortices are nucleated, then changing to a second value of $\Omega$, one can attain a very different angular momentum compared to that achieved when the second $\Omega$ is retained throughout. Furthermore, we have shown that simulations with different initial conditions, corresponding to when vortices are already present in the condensate, also lead to different final angular momenta, similar to behavior found in the experiment of Ref. [18]. Using a hydrodynamic model we have demonstrated that this effect is due to a shift of the resonant frequency for the excitation of quadrupole collective modes when the condensate is already in rotation. We also show that the angular momentum response to a linear ramp of the trap rotation frequency is radically different depending on whether the ramp is increasing or decreasing, revealing the existence of hysteresis phenomena in rotating condensates.

We conclude by noting that these processes should be readily observable in present experiments where vortices have been nucleated by stirring the condensate (e.g. Refs. 2, 4, 6]), although the dissipation arising from the presence of a non-condensed cloud may influence the angular momentum eventually achieved over longer time scales. Assessing the importance of this contribution requires a more sophisticated model that includes finite temperature 
effects [35, 36], and will be left to future work.

\section{Acknowledgments}

Financial support for this research was provided by the Ministero dell'Instruzione, dell'Universitá e della Ricerca, and the Engineering and Physical Sciences Research Council. We gratefully acknowledge useful discussions with C. Adams, A. Fetter, N. Parker and N. Proukakis.

[1] R. J. Donnelly, Quantized Vortices in Helium II (Cambridge University Press, 1991)

[2] K. W. Madison, F. Chevy, W. Wohlleben, and J. Dalibard, Phys. Rev. Lett. 84, 806 (2000).

[3] F. Chevy, K. W. Madison, and J. Dalibard, Phys. Rev. Lett. 85, 2223 (2000).

[4] J. R. Abo-Shaeer, C. Raman, J. M. Vogels, and W. Ketterle, Science 292, 476 (2001).

[5] C. Raman, J. R. Abo-Shaeer, J. M. Vogels, K. Xu, and W. Ketterle, Phys. Rev. Lett. 87, $210402(2001)$.

[6] E. Hodby, G. Hechenblaikner, S. A. Hopkins, O. M. Maragó, and C. J. Foot, Phys. Rev. Lett. 88, 010405 (2002).

[7] P. C. Haljan, I. Coddington, P. Engels, and E. A. Cornell, Phys. Rev. Lett. 87, 210403 (2001).

[8] K. W. Madison, F. Chevy, V. Bretin, and J. Dalibard, Phys. Rev. Lett. 86, 4443 (2001).

[9] M. W. Zwierlein, J. R. Abo-Shaeer, A. Schirotzek, C. H. Schunck, and W. Ketterle, Nature 435, 1047 (2005).

[10] F. Dalfovo and S. Stringari, Phys. Rev. A 53, 2477 (1996).

[11] A. L. Fetter and A. A. Svidzinsky, J. Phys.: Condens. Matter 13, R135 (2001).

[12] F. Dalfovo and S. Stringari, Phys. Rev. A 63, 011601(R) (2000).

[13] M. Krämer, L. Pitaevskii, S. Stringari, and F. Zambelli, Laser Physics 12, 113 (2002).

[14] J. J. García-Ripoll and V. M. Pérez-García, Phys. Rev. A 63, 041603(R) (2001).

[15] R. M. Packard and T. M. Sanders, Phys. Rev. A 6, 799 (1972).

[16] P. Mathieu, J. C. Marechal, and Y. Simon, Phys. Rev. B 22, 4293 (1980).

[17] C. A. Jones, K. B. Khan, C. F. Barenghi, and K. L. Henderson, Phys. Rev. B 51, 16174 (1995). 
[18] V. Bretin, S. Stock, Y. Seurin, and J. Dalibard, Phys. Rev. Lett. 92, 050403 (2004).

[19] D. L. Feder, A. A. Svidzinsky, A. L. Fetter, and C. W. Clark, Phys. Rev. Lett. 86, 564 (2001).

[20] E. Lundh, J. -P. Martikainen, and K. -A. Suominen, Phys. Rev. A 67, 063604 (2003).

[21] K. Kasamatsu, M. Tsubota, and M. Ueda, Phys. Rev. A 67, 033610 (2003).

[22] C. Lobo, A. Sinatra, and Y. Castin, Phys. Rev. Lett. 92, 020403 (2004).

[23] N. G. Parker and C. S. Adams, Phys. Rev. Lett. 95, 145301 (2005).

[24] N. G. Parker and C. S. Adams, J. Phys. B: At. Mol. Opt. Phys. 39, 43 (2006).

[25] M. Cozzini and S. Stringari, Phys. Rev. A 67, 041602(R) (2003).

[26] D. Guéry-Odelin, Phys. Rev. A 62, 033607 (2000).

[27] M. Tsubota, K. Kasamatsu, and M. Ueda, Phys. Rev. A 65, 023603 (2002).

[28] A. A. Penckwitt, R. J. Ballagh, and C. W. Gardiner, Phys. Rev. Lett. 89, 260402 (2002).

[29] F. Zambelli and S. Stringari, Phys. Rev. A 63, 033602 (2001).

[30] J. J. García-Ripoll and V. M. Pérez-García, Phys. Rev. A 64, 013602 (2001).

[31] Note that for $\sqrt{1-\epsilon}<\Omega<\sqrt{1+\epsilon}$ motion of the condensate center-of-mass is dynamically unstable [32], such that its displacement from the center of the trap grows exponentially in time. This precludes simulations in the range $0.95<\Omega<1.05$ for $\Omega_{0}=0.5$, since the small (but non-zero) initial displacement of the center-of-mass leads to the condensate rapidly spiralling out of the numerical grid. On the other hand, for $\Omega_{0}=0$ the initial displacement is very close to zero, so that the instability is less of a problem over applicable timescales, and simulations are possible in this range.

[32] P. Rosenbusch, D. S. Petrov, S. Sinha, F. Chevy, V. Bretin, Y. Castin, G. Shlyapnikov, and J. Dalibard, Phys. Rev. Lett. 88, 250403 (2002).

[33] Eqs. (6) and (7) can be easily extended to three dimensions by including the additional parameters $a_{z}$ and $b_{z}$, as in 25]. Similar results are found, further verifying that the 2D approach taken here is reasonable.

[34] W. H. Press, S. A. Teukolsky, W. T. Vetterling, and B. P. Flannery, Numerical Recipes in Fortran (Cambridge University Press, 1992)

[35] E. Zaremba, T. Nikuni, and A. Griffin, J. Low. Temp. Phys. 116, 277 (1999).

[36] B. Jackson and E. Zaremba, Phys. Rev. A 66, 033606 (2002). 